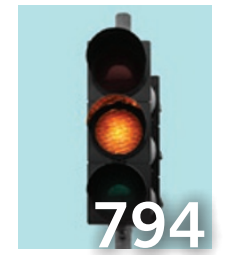 \\ Waiting game: \\ A case study finds \\ EU rules slow trial \\ approvals

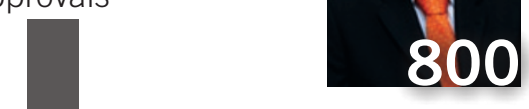 \\ Best practice: \\ Otmar Kloiber tells \\ why the Declaration \\ of Helsinki matters

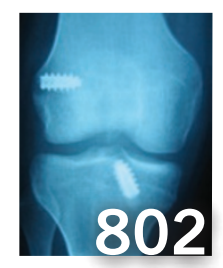 \\ On the mend: \\ Researchers try \\ radical therapies \\ for sports injuries \\ Mystery of preterm birth prompts search for better models
}

As the number of preterm births continues to climb, experts say they need better research tools to deliver answers to this puzzling problem in pregnancy. Doctors and researchers made the case for launching new and bigger studies on this subject at a two-day conference on the prevention of preterm births, hosted in Rockville, Maryland during June by the acting US surgeon general, Steven Galson.

About half a million—one in eight—babies born in the US are preterm, meaning they are delivered at least three weeks early (less than 37

weeks' gestation). The percentage of preterm births has increased by $30 \%$ since the early 1980s and shows no signs of abating, according to a 2006 report by the Institute of Medicine. Crucially, even though many 'preemies' survive the initial medical complications of premature birth, they sometimes suffer permanent health consequences, including learning disabilities, cerebral palsy and impaired vision.

At the recent meeting in Rockville, experts acknowledged the need to know the biological mechanisms behind preterm birth. "Without doing that, you cannot prevent it," says Catherine Spong from the US National Institute of Child Health and Human Development in Rockville.

To isolate the causes of preterm birth, scientists must first identify better animal and human tissue models of it, says George Saade, who co-chaired the conference's biomedical research committee with Spong. "We also need noninvasive methods to investigate uterine and cervical function and placental and fetal development," adds Saade, who also directs the division of maternal-fetal medicine at the University of Texas Medical Branch in Galveston.

Stella Kourembanas, chief of the newborn medicine department at the Children's Hospital in Boston, could not agree more. But she adds that special emphasis should be directed toward understanding how the placenta can malfunction and force a premature delivery, as in the case of pre-eclampsia, a common complication characterized by high blood pressure in the mother. "Placental biology—I think it is the key to understanding [preterm birth]," Kourembanas explains.

Also top on the research agenda is a long-term study that would ideally track several thousand

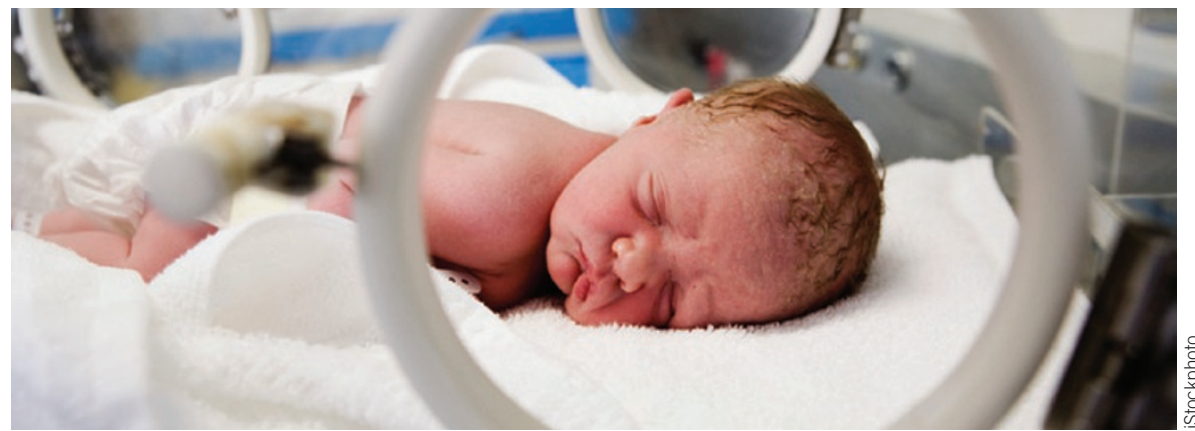

A window to the future: A better grasp on placental function might prevent some preterm births

women before, during and several years after their first pregnancy, according to Saade. This type of investigation would enable scientists to collect biological samples and other health data that could be used to identify biomarkers along with genetic and environmental risk factors.

Such information would be invaluable, Kourembanas notes, particularly for understanding the preterm births that occur later in term, between 34 and 37 weeks' gestation. Until now, most studies have centered on the younger preemies, who tend to have more severe health problems but account for a shrinking proportion of preterm births.

Experts also stress that there must be greater investment in centers where clinicians, basic science researchers and epidemiologists all work together. The mysteries surrounding preterm births - why black women in the US are affected more often, for example — can only be solved with a multidisciplinary approach, says Michael Kramer of McGill University in Montreal and co-chair of the conference's epidemiological research group. "This is a very complex problem that needs to be understood from the societal level to the molecular level," he says.

Addressing the preterm birth issue will require an infusion of funding, scientists in the field say. Few researchers have elected to pursue this line of research because grants are so scarce, says Saade. "The government needs to allocate more money toward this problem," he says.

"If we have support for this," Kourembanas says, "I think it will revolutionize prevention and treatment."

Coco Ballantyne, New York

\section{One egg is best}

For some couples struggling with infertility, the idea of having twins or triplets with the help of in vitro fertilization (IVF) may be appealing. But multiple pregnancies are risky - mothers are more likely to suffer medical complications such as gestational diabetes, and babies are often born preterm.

In the UK, $24 \%$ of all IVF pregnancies involve twins or triplets. A group of concerned doctors, nurses and patient groups have banded with the UK's Human Fertilisation and Embryology Authority to develop a national strategy to reduce multiple pregnancies from IVF to ten percent within three years. On 26 June, they unveiled plans for a series of professional workshops, the launch of an informational website and a new set of best-practice guidelines.

IVF increases the chance of a multiple pregnancy because doctors often transfer more than one embryo to the uterus at a time. Fertility experts need to identify the women at greatest risk for multiple pregnancies - typically healthy women under the age of 37- and provide them with single rather than multiple embryo transfers, explains Rachel Cutting, an embryologist on the executive committee of the Association of Clinical Embryologists in Britain.

Coco Ballantyne, New York 